\title{
ГРУППОВОЙ ПОДХОД В ДИНАМИКЕ МНОГОСПИНОВЫХ СИСТЕМ. VII
}

\author{
(Представлена Э. Липпмаа)
}

В первой статье этой серии [1] в пространстве эрмитовых операторов рассматривалось два типа базисов - $A$-базис и $I$-базис. Первый из них описывал постоянную часть гамильтониана изучаемой системы, используя язык, близкий к языку матричной механики. На этом языке удобно формулировать общие закономерности квантовой динамики, в том числе и резонансные свойства. При этом, однако, конкретная природа системы остается неопределенной. Такой абстрактной динамике трехуровневой системы была, в частности, посвящена работа [ $\left.{ }^{2}\right]$.

В данной статье трехуровневая система считается ядерным спином 1, взаимодействующим с внешними (постоянными и переменными) магнитными и неоднородными электрическими полями. Тем самым содержание работы относится к областям ядерного магнитного резонанса (ЯМР), ядерного квадрупольного резонанса (ЯКР) и ядерного акустического резснанса (ЯАР), но охватывает более широкий круг условий опыта, чем встречается на практике. Результаты абстрактной динамики $\left[{ }^{2}\right]$ интерпретируются на языке $I$-базиса, что позволяет установить связь между квантовыми и классическими величинами, описывающими внешние поля и наблюдаемые сигналы.

\section{1. Динамика ядерного спина 1}

11.1. Қлассические и кв антовы е величины. Рассмотрим динамику больцмановского ансамбля, состоящего из ядерных спинов 1 . Основные классические и квантовые величины, необходимые для описания этой динамики, представлены на схеме (11.1)

$$
\begin{aligned}
& \vec{B}(t) \\
& \mathbf{U}(t)
\end{aligned} \leftrightarrow H(t) \rightarrow \varrho(t) \leftrightarrow \begin{aligned}
& \vec{M}(t) \\
& e \mathbf{Q}(t)
\end{aligned}
$$

Действующие на ядра классические поля - магнитное $\vec{B}(t)$ и характеризуемое тензором $\mathbf{U}(t)$ неоднородное электрическое - определяют гамильтониан $H(t)$. Гамильтониан и начальное состояние $\varrho(0)$ предопределяют временное изменение оператора плотности $\mathrm{Q}(t)$. Последнему, в свою очередь, однозначно соответствует временное изменение классических выходных величин: среднего ядерного магнитного момента $\vec{M}(t)$ и среднего ядерного квадрупольного момента $е \mathbf{Q}(t)$.

В работе [2] соответствие между $H(t)$ и $\mathrm{g}(t)$ исследовалось в рамках динамики абстрактной трехуровневой системы. В данной работе полученные результаты прилагаются к динамике спина 1 для изучения: а) соответствия между классическими и квантовыми величинами, 
представленными на схеме $(11.1)$, б) соотношения между абстрактной и спиновой динамиками.

Оба отмеченные на схеме (11.1) взаимно-однозначные соответствия между классическими и квантовыми величинами основываются на изоморфизме их линейных алгебр. Как те, так и другие являются (приводимыми) евклидовыми тензорами второго ранга, определенными относительно (декартового) лабораторного репера $\vec{a}_{j}(j=x, y, z)\left[{ }^{3}\right]$. Отличительная черта квантовых величин $H(t), \mathrm{Q}(t)$ в том, что они к тому же и эрмитовые операторы, действующие в 3-мерном унитарном пространстве C чистых квантовых состояний. Линейные операторы над С образуют 9-мерное унитарное пространство О. Из последнего можно выделить 9-мерное евклидовое пространство Н всех эрмитовых операторов [ $\left.{ }^{1}\right]$. Алгебра в Н является не только алгеброй тензоров, но и алгеброй Ли $\mathbf{u}_{3}{ }^{0}\left[{ }^{4,2}\right]$.

Сопоставляя каждому $\vec{a}_{j}$ спиновый оператор $I_{j}$

$$
\vec{a}_{j} \in \mathbf{V} \leftrightarrow I_{j} \in \mathbf{G}_{Z}^{0} \subset \mathbf{H},
$$

устанавливаем тем самым соответствие между обыкновенным векторным пространством $\mathbf{V}$ и его алгеброй Ли $\mathbf{o}_{3}{ }^{0}$, с одной стороны, и натянутым на базис $I_{j} 3$-мерным подпространством $\mathbf{G}_{Z}{ }^{0} \subset \mathbf{H}$ и его алгеброй Ли $\mathbf{u}_{2}{ }^{0}$, с другой стороны. При этом векторному произведению $\left[\overrightarrow{a_{j}}, \overrightarrow{a_{k}}\right]$ будет соответствовать коммутатор $-i\left[I_{j}, I_{k}\right]$. В нормах базисных векторов обоих пространств имеется различие: орты $\vec{a}_{j}$ образуют ортонормированную систему, а

$$
\left(I_{j}, I_{k}\right)=\operatorname{tr} I_{j} I_{k}=2 \delta_{j k} .
$$

Пусть собственные векторы $a_{m}(m=1,2,3)$ эрмитового оператора $I_{z}$

$$
I_{z} a_{m}=\mu_{m} a_{m},
$$

где $\mu_{1}=1, \mu_{2}=0, \mu_{3}=-1$, служат в качестве стандартного $a_{m}$-базиса пространства $\mathbf{C}\left[{ }^{5}\right]$. Соответствующие им $A$-базисы пространств $\mathbf{O}$ и $\mathbf{H}$ $\left[{ }^{1,2}\right]$ будем тоже называть стандартными.

Путем составления прямых произведений векторов $\overrightarrow{a_{j}}$ образуем ортонормированный базис $\mathrm{a}_{j k}(j, k=x, y, z)$ 9-мерного пространства $\mathbf{V} \times \mathbf{V}$ приводимых евклидовых тензоров второго ранга. Базису $\mathbf{a}_{j k}$ сопоставляем аналогичный базис $I_{j k}$ пространства $\mathbf{O}$

$$
\mathbf{a}_{j k}=\overrightarrow{a_{j}} \times \overrightarrow{a_{k}} \leftrightarrow I_{j k}=I_{j} I_{k} .
$$

Однако матричные произведения $I_{j k}$ не являются эрмитовыми операторами и не составляют ортогонального базиса. Поэтому в качестве соответствующих друг другу ортогональных базисов пространств $\mathbf{V} \times \mathbf{V}$ и H выбираем базисы, составленные из неприводимых тензоров $\mathbf{a}_{q}^{(l)}$ и $I_{q}^{(l)}(l=0,1,2)$ соответственно. Они суть действительные аналоги распространенных в физике комплексных неприводимых тензоров [ $\left.{ }^{3,5}\right]$.

Назовем базис пространства $\mathbf{H}$, составленный из неприводимых спиновых тензоров $I_{q}^{(l)}, I$-базисом. Выражения операторов $I_{q}^{(l)}$ на языке операторов $I_{j k}$ и на языке стандартного $A$-базиса приведены в табл. 1. Заменяя там $I_{j k}$ на $\mathbf{a}_{j k}$, получим выражения базисных тензоров $\mathbf{a}_{q}^{\left({ }^{(l)}\right.}$ через $\mathbf{a}_{j k}$ : 


\section{Выражения операторов $\underset{q}{I_{q}^{(l)}} \quad I$-базиса пространства $\mathbf{H}$}

\begin{tabular}{l|l|l}
\hline $\begin{array}{c}I_{q}^{(l)} \\
I_{0}^{(0)}\end{array}$ & На языке операторов $I_{j k}$ & На языке стандартного $A$-базиса \\
\hline$I_{1}^{(1)}$ & $I_{x}=\sum_{j} I_{j j}$ & $2\left(A_{11}+A_{22}+A_{33}\right)$ \\
$I_{2}^{(1)}$ & $I_{y}$ & $X_{1}(13)=\sqrt{2}\left(X_{12}+X_{23}\right)$ \\
$I_{3}^{(1)}$ & $I_{z}$ & $Y_{1}(13)=\sqrt{2}\left(Y_{12}+Y_{23}\right)$ \\
$I_{0}^{(2)}$ & $(1 / 3)\left(3 I_{z z}-I^{2}\right)$ & $2 Z_{13}$ \\
$I_{1}^{(2)}$ & $I_{x x}-I_{y y}$ & $Q_{13}$ \\
$I_{2}^{(2)}$ & $I_{x y}+I_{y x}$ & $2 X_{13}$ \\
$I_{3}^{(2)}$ & $I_{z x}+I_{x z}$ & $2 Y_{13}$ \\
$I_{4}^{(2)}$ & $I_{y z}+I_{z y}$ & $X_{2}(13)=\sqrt{2}\left(X_{12}-X_{23}\right)$ \\
& & $Y_{2}(13)=\sqrt{2}\left(X_{12}-Y_{23}\right)$ \\
& \multicolumn{2}{c}{}
\end{tabular}

Оба базиса $\mathrm{a}_{q}^{(l)}$ и $I_{q}^{(l)}$ не являются нормированными. Скалярные произведения базисных тензоров равны

$$
\begin{gathered}
\left(\mathbf{a}_{q}^{(l)}, \mathbf{a}_{q}^{(l)}\right)=\left(I_{q}^{(l)}, I_{q}^{(l)}\right)=2 \text { при } l \neq 0, q \neq 0, \\
\left(\mathbf{a}_{0}^{(2)}, \mathbf{a}_{0}^{(2)}\right)=\left(I_{0}^{(2)}, I_{0}^{(2)}\right)=2 / 3 .
\end{gathered}
$$

Спиновые тензоры $I_{q}^{(l)}$ с $l=1,2$ составляют базис 8-мерного кольца Ли $\mathbf{u}_{3}{ }^{0}$. Кроме $\varrho(t)$, все нужные нам эрмитовые операторы будут элементами $\mathbf{u}_{3}{ }^{0}$.

При вращениях лабораторного репера

$$
\overrightarrow{e_{j}}=\Omega \overrightarrow{a_{j}}
$$

базисные векторы $\mathbf{a}_{q}^{(l)}$ и $I_{q}^{(l)}$ с одинаковым значением $l$ преобразуются по тензорному представлению группы $\mathbf{O}_{3}\left[{ }^{6}\right]$ как неприводимые тензоры порядка $l$. Разложение $\mathbf{u}_{3}{ }^{0}$ на неприводимые по $\mathbf{O}_{3}$ подпространства имеет вид

$$
\mathbf{u}_{3}^{0}=\mathbf{G}_{z}^{0}+\mathbf{H}_{Q},
$$

где 5-мерное $\mathbf{H}_{Q}$ натянуто на $I_{q}{ }^{(2)}$.

Любой приводимый евклидовый тензор $\mathbf{L} \in \mathbf{V} \times \mathbf{V}$ разлагается согласно

$$
\mathbf{L}=\sum_{j} \sum_{k} L_{j k} \mathbf{a}_{j k}=\sum_{l} \sum_{q} L_{q}^{(l)} \mathbf{a}_{q}^{(l)},
$$

где связь компонент $L_{q}{ }^{(l)}$ и $L_{j k}$ задается формулами

$$
\begin{aligned}
& L_{0}^{(0)}=(1 / 3) \sum_{j} L_{j j}, \\
& L_{1}^{(1)}=(1 / 2)\left(L_{y z}-L_{z y}\right)=L_{x}, \\
& L_{2}^{(1)}=(1 / 2)\left(L_{z x}-L_{x z}\right)=L_{y}, \\
& L_{3}^{(1)}=(1 / 2)\left(L_{x y}-L_{y x}\right)=L_{z},
\end{aligned}
$$




$$
\begin{aligned}
& L_{0}^{(2)}=(1 / 2)\left(3 L_{z z}-\sum_{j} L_{j j}\right), \\
& L_{1}^{(2)}=(1 / 2)\left(L_{x x}-L_{y y}\right) \\
& L_{2}^{(2)}=(1 / 2)\left(L_{x y}+L_{y x}\right) \\
& L_{3}^{(2)}=(1 / 2)\left(L_{z x}+L_{x z}\right) \\
& L_{4}^{(2)}=(1 / 2)\left(L_{y z}+L_{z y}\right) .
\end{aligned}
$$

Классические величины на схеме (11.1) суть элементы пространства V X.V. В частности, аксиальный вектор

$$
\vec{B}(t)=\sum_{j} B_{j}(t) \vec{a}_{j}
$$

относится к неприводимым тензорам первого порядка. Взаимодействие неоднородного электрического поля

$$
\vec{E}(t)=-\operatorname{grad} U(t)
$$

с ядерным квадрупольным моментом описывается значениями вторых производных потенциала, вроде

$$
U_{x y}(t)=\frac{\partial^{2} U(t)}{\partial x \partial y},
$$

в точке положения ядра $\left[{ }^{7,8}\right]$. Величины типа (11.17) образуют симметричный тензор

$$
\mathbf{U}(t)=\sum_{j} \sum_{k} U_{j k}(t) \mathbf{a}_{j k}=\sum_{q} U_{q}^{(2)}(t) \mathbf{a}_{q}^{(2)} .
$$

Так как поле (11.16) является внешним относительно ядра, то выполняется уравнение Лапласа

$$
\sum_{j} U_{j j}(t)=0
$$

Нтак, тензор (11.18) неприводимый.

Квантовые величины на схеме (11.1) суть элементы пространства $\mathbf{H}$. Таковыми являются также операторы компонент ядерного магнитного момента

$$
M_{j}=\gamma \hbar I_{j}
$$

и ядерного электрического квадрупольного момента

$$
\begin{aligned}
& e Q_{j k}=e Q I_{j k}, \\
& e Q_{q}^{(2)}=e Q I_{q}^{(2)} .
\end{aligned}
$$

В вышеприведенных формулах $\gamma$ - ядерное гиромагнитное отношение, $e Q$ - скалярный ядерный квадрупольный момент $\left[{ }^{7}\right]$.

По принципу классической аналогии, гамильтониан ядра, взаимодействующий с полями (11.15), (11.16),

$$
H(t)=H_{Z}(t)+H_{Q}(t)
$$

состоит из зеемановского и квадрупольного членов:

2 ENSV TA Toimetised $F^{*} M 31979$ 


$$
\begin{gathered}
\hbar H_{Z}(t)=-\sum_{j} B_{j}(t) M_{j}=-\hbar \sum_{j} \omega_{j}(t) I_{j} \in \mathbf{G}_{Z}^{0}, \\
\hbar H_{Q}(t)=\frac{1}{2} \sum_{j} \sum_{k} U_{j k}(t) Q_{j k}=\frac{1}{2} e Q \sum_{j} \sum_{k} U_{j k}(t) I_{j k} \in \mathbf{H}_{Q} .
\end{gathered}
$$

В формулу (11.24) введены ларморовые частоты

$$
\omega_{j}(t)=\gamma B_{j}(t)
$$

в предположении, что $\gamma>0$. Если к тому же в (11.25) заменить приводимые тензоры на неприводимые, то гамильтониан (11.23) примет вид

$$
H(t)=-\sum_{j} \omega_{j}(t) I_{j}+\frac{e Q}{2 \hbar} \sum_{q} U_{q}^{(2)}(t) I_{q}^{(2)} .
$$

Если оператор плотности тоже представить его неприводимыми компонентами на I-базисе

$$
\varrho(t)=\sum_{l} \sum_{q} \varrho_{q}^{(D)}(t) I_{q}^{(D)},
$$

то наблюдаемые компоненты среднего (по ансамблю) магнитного момента

$$
\vec{M}(t)=\sum_{j}\left\langle M_{j}(t)\right\rangle \vec{a}_{j}
$$

и среднего электрического квадрупольного момента

$$
e \mathbf{Q}(t)=\sum_{q}\left\langle e Q_{q}^{(2)}(t)\right\rangle \mathbf{a}_{q}^{(2)}
$$

вычисляются по формулам

$$
\begin{gathered}
\left\langle M_{j}(t)\right\rangle=\left(\varrho(t), M_{j}\right)=2 \gamma{ }^{\hbar} \varrho_{j}^{(1)}(t), \\
\left\langle e Q_{q}^{(2)}(t)\right\rangle=e Q\left(\varrho(t), I_{q}^{(2)}\right)=e Q\left(I_{q}^{(2)}, I_{q}^{(2)}\right) \varrho_{q}^{(2)}(t) .
\end{gathered}
$$

При изложении абстрактной динамики трехуровневой системы $\left[{ }^{2}\right]$ важное значение имели представления алгебры Ли $\mathbf{u}_{2}{ }^{0}$, примыкающие к $A$-базису. Базисные операторы тех представлений типа $\mathbf{G}^{0}(\mathrm{mn})$, которые соответствуют стандартному $A$-базису, приведены в табл. 2 . На основе табл. 1 и 2 , ' а также формул п. $10.3\left[{ }^{2}\right]$ нетрудно перевести на язык $I$-базиса и базисы представлений типа $\mathbf{G}^{0}(m k, k n)$. В частности, по данным табл. 1 , подкольцо $\mathbf{G}_{z^{0}}$ относится к типу $\mathbf{G}^{0}(12,23)$.

Подведем некоторые итоги. Симметрия $\mathbf{O}_{3}$ (пространственные вращения и инверсия) основного состояния ядра и малые по сравнению с

Таблица 2

Базисы подколец типа $\mathbf{G}^{0}(m n)$, примыкающие к стандартному $A$-базису

\begin{tabular}{c|l|c|c}
\hline$W_{m n}$ & $\mathbf{G}^{0}(13)$ & $\mathbf{G}^{0}(12)$ & $\mathbf{G}^{0}(23)$ \\
\hline$Q_{m n}$ & $I_{0}^{(2)}$ & $(1 / 2)\left(I_{z}-I_{0}^{(2)}\right)$ & $-(1 / 2)\left(I_{z}+I_{0}^{(2)}\right)$ \\
$Z_{m n}$ & $(1 / 2) I_{z}$ & $(1 / 4)\left(I_{z}+3 I_{0}^{(2)}\right)$ & $(1 / 4)\left(I_{z}-3 I_{0}^{(2)}\right)$ \\
$X_{m n}$ & $(1 / 2) I_{1}^{(2)}$ & $\left(1 / 2 \sqrt{2)}\left(I_{x}+I_{3}^{(2)}\right)\right.$ & $(1 / 2 \sqrt{2})\left(I_{x}-I_{3}^{(2)}\right)$ \\
$Y_{m n}$ & $(1 / 2) I_{2}^{(2)}$ & $\left(1 / 2 \sqrt{2)}\left(I_{y}+I_{4}^{(2)}\right)\right.$ & $(1 / 2 \sqrt{2})\left(I_{y}-I_{4}^{(2)}\right)$
\end{tabular}


межатомными расстояниями его размеры (словом - структура вещества) делают правомерным разложение классических величин на неприводимые компоненты представления группы $\mathbf{O}_{3}$ по схеме (11.1). Этим же оправдывается аналогичное разложение квантовых величин (11.10), достигаемое введением I-базиса.

На преимущества $A$-базиса перед $I$-базисом при установлении соответствия между $H(t)$ и $\varrho(t)$ указывалось неоднократно. Преимущества $A$-базиса усугубляются тем, что на практике

$$
\begin{gathered}
\vec{B}(t)=\vec{B}_{0}+\vec{B}_{E}(t), \\
\mathbf{U}(t)=\mathbf{U}_{0}+\mathbf{U}_{E}(t),
\end{gathered}
$$

причем переменные составляющие слабы по сравнению с постоянными. В силу (11.33) и (11.34) гамильтониан (11.27) может быть также разложен на статическую часть $H_{0}$ и возбуждение $H_{E}(t)$ :

$$
H(t)=H_{0}+H_{E}(t) .
$$

Как та, так и другая могут (в принципе) состоять из зеемановского и квадрупольного взаимодействий. В частности, будем говорить о зеемановском $H_{E Z}(t)$ 'и кквадрупольном $H_{E Q}(t)$ возбуждениях. Тип возбуждения зависит от выбранных условий опыта. Статический же гамильтониан $H_{0}$ будем считать характеристикой спиновой системы.

Подходящим для описания динамики $A$-базисом оказывается тот, который построен исходя из собственных векторов оператора $H_{0}$ изучаемой системы (системный $A$-базис). Стандартный $A$-базис является системным только в случае т. н. стандартных систем, обладающих гамильтонианами типа

$$
H_{0}=-\omega_{Z} I_{z}+(3 / 4) \omega_{Q} I_{0}^{(2)} \text {, }
$$

где

$$
\omega_{Q}=e Q U_{z z} / \hbar
$$

- постоянная квадрупольного взаимодействия $\left[{ }^{7,8}\right]$. Соответствующие гамильтониану (11.36) постоянные поля

$$
\begin{aligned}
& \vec{B}_{0}=B_{0} \vec{a}_{z}, \\
& \mathbf{U}_{0}=(3 / 2) U_{z z} \mathbf{a}_{0}^{(2)}
\end{aligned}
$$

обладают осью симметрии по $\vec{a}_{z}$.

Данные табл. 2 показывают, что только в случае $H(t) \in \mathbf{G}_{z}{ }^{0}$ (в случае чистого магнитного резонанса) взаимодействия определенного типа (зеемановские) обусловливают замкнутую поддинамику, описываемую некоторым представлением $\mathbf{G}_{Z}$ группы $\mathbf{u}_{2}$. Все другие поддинамики связаны со «смешанным» типом взаимодействий. Динамической группы, которая бы описывала чистый квадрупольный резонанс, не существует. Применение только зеемановского возбуждения (ЯМР, ЯКР, но не ЯАР) не позволяет реализовать все движения $\mathrm{Q}(t)$, допускаемые динамической группой $\mathbf{u}_{3}$.

Чтобы воспользоваться результатами абстрактной динамики $\left[{ }^{2}\right]$ и систематизировать динамику по структуре алгебры Ли $\mathbf{u}_{3}{ }^{0}$ (а не по типам взаимодействий), необходимо установить соответствие между $I$-базисом и системными $A$-базисами. Сделаем это с помощью понятия эквивалентных динамик $\left[{ }^{1}\right]$. 
11.2. Абстрактная и спиновая динамики. Унитарные операторы $T \in \mathbf{u}_{3}$ могут быть использованы в целях классификации статических гамильтонианов (тем самым и спиновых систем). Применяя отныне символ $H_{0}$ только для гамнльтонианов вида $(11.36)$, имеем в случае всех других систем

$$
H_{T}=T H_{0} T^{-1} \text {. }
$$

Қаждый гамильтониан (11.40) характеризуется: а) спектром своих собственных значений (два параметра вроде $\left.\omega_{Z}, \omega_{Q}\right)$, б) унитарной ориентацией $T$ своих собственных векторов (6 параметров Мойнихена $\left.\left[{ }^{2}\right]\right)$. Операторы $H_{T}$, различающиеся только унитарной ориентацией, образуют класс эквивалентных операторов (спектральный класс систем). Представители всех классов имеются среди стандартных систем, т, е. в подпространстве $\mathbf{H}_{A}{ }^{0}$ стандартного $A$-базиса.

Символы $Q_{m n}, Z_{m n}, X_{m n}, Y_{m n}$ будем отныне относить только к стандартному $A$-базису, а в качестве общего символа этих базисных операторов возьмем обозначение $W_{m n}$. Тогда любой другой системный $A$-базис, соответствующий гамильтониану вида (11.40), получается ортогональным преобразованием стандартного $A$-базиса

$$
W_{m n}(T)=\mathfrak{I} W_{m n}=T W_{m n} T^{-1} .
$$

В частности, операторы $Q_{m n}(T), Z_{m n}(T)$ образуют базис 2-мерного подпространства $\mathbf{H}_{T}^{0}$, содержащего все гамильтонианы (11.40).

Абстрактная динамика $\left[{ }^{2}\right]$ применима в равной мере ко всем системным $A$-базисам. Если гамильтониану стандартной системы

$$
H(t)=\sum_{m} \sum_{<n} \omega_{m n}(t) W_{m n}
$$

соответствует семейство траекторий

$$
\mathrm{Q}(t)=\sum_{m<n} \sum_{m n}(t) W_{m n},
$$

то семейство траекторий

$$
\varrho_{T}(t)=\sum_{m<n} \sum_{<n n}(t) W_{m n}(T)
$$

соответствует гамильтониану

$$
H_{T}(t)=\sum_{m<n} \sum_{m n}(t) W_{m n}(T)
$$

некоторой. эквивалентной системы (того же спектрального класса́), имеющей статический гамильтониан вида (11.40).

Чтобы установить, какие поля $\vec{B}(t), \mathbf{U}(t)$ приводят к гамильтониану (11.45) и какие наблюдаемые $\vec{M}(t)$ и $е \mathbf{Q}(t)$ соответствуют оператору плотности (11.44), необходим перевод всличин (11.44), (11.45) на язык I-базиса. В результате такого перевода $H_{T}(t)$ принимает общий вид

$$
H_{T}(t)=\sum_{l} \sum_{q} \omega_{q}^{(l)}(t) I_{q}^{(l)},
$$

а более конкретно - вид (11.27). Оператор же $\varrho_{T}(t)$ будет задаваться выражением (11.28).

1. Зависимость $\omega_{q}^{(l)}(t)$ и $\varrho q^{(l)}(t)$ от $\omega_{m n}(t)$ и $\varrho m n(t)$ соответственно устанавливается с помощью матриц супероператоров $\mathcal{I}$ и $\mathbb{2}:$ 


$$
W_{m n}=\sum_{l} \sum_{q} \Omega_{l q, m n} I_{q}^{(l)}
$$

О супероператоре $\mathfrak{I}$ речь шла в п. $10.1\left[^{2}\right]$. Матрицу же супероператора $\&$ нетрудно получить на основе табл. 1. На базе этих данных величины $\omega_{q}^{(l)}(t)$ и $q^{(l)}(t)$ вычисляются по формулам

$$
\begin{aligned}
& \omega_{q}^{(l)}(t)=\sum_{m<n} \sum_{(\mathcal{L})_{l q, m n} \omega_{m n}(t),} \\
& \mathrm{Q}_{q}^{(l)}(t)=\sum_{m<n} \sum_{(\mathcal{L})_{l q, m n} \mathrm{Q}_{m n}(t) .}
\end{aligned}
$$

\section{Л ИТ Е Р А Т У Р А}

1. С и н и в е э В., Изв. АН ЭССР, Физ. Матем., 24, № :1, 35-48 (1975).

2. С и н и в е э В., Изв. АН ЭССР, Фнз. Матем., 28, № 2, 115-123 (1979).

3. Fa no, U., Racah, G., Irreducible tensorial sets, Academic Press, New York, 1959.

4. B o erner, H., Representations of groups, North-Holland, Amsterdam, 1963.

5. Messiah, A., Quantum mechanics, 2, North-Holland, Amsterdam, 1964.

6. Г ельфанд И. М., Мин лос Р. А., Ша п и ро 3. Я., Представления группы вращений и группы Лоренца, их применения, М., Физматгиз, 1958 ,

7. Ко п фе р м а н Г., Ядерные моменты, М., Изд-во иностр. лит., 1960.

8. Г ре чишкин В. С., Ядерные квадрупольные взаимодействия в твердых телах, М., «Наука», 1973.

Ннститут кибернетики

Академии наук Эстонской ССР
Поступила в редакцию

$27 /$ IV 1979

\section{SINIVEE}

\section{RUHMADE TEOORIA RAKENDAMISEST MITMESPINNISUSTEEMIDE DUNAAMIKA UURIMISEKS. VII}

Artiklis on käsitletud 1-spinnilise tuuma kvantdünaamikat.

\section{SINIVEE}

\section{GROUP APPROACH IN DYNAMICS OF MANY-SPIN SYSTEMS. VII}

The dynamics of a nuclear spin interacting with external time-dependent magnetic and inhomogeneous electric fields is considered as an application of the previously developed theory of an abstract three-level quantum system. Eq. (11.1) gives the classical quantities and quantum mechanical operators needed. The correspondence between both kinds of quantities is based on isomorphic tensorial algebras. The $I$-basis (Table 1) helps to express this relationship. On the other hand, the abstract dynamics is described in terms of an operator basis (the $A$-basis) which is originated from matrix algebra and is specified by the spin system under study. The relationship between these two modes of description is given by transformations (11.48), (11.49). 